\title{
Evaluating the use of benzodiazepines following recent bereavement
}

\author{
JAMES WARNER, CHRIS METCALFE and MICHAEL KING
}

\author{
Background There is no evidence to \\ support current advice not to use \\ benzodiazepines after bereavement.
}

\begin{abstract}
Aims To determine the role of benzodiazepines in the management of bereavement.
\end{abstract}

\begin{abstract}
Method We conducted a randomised, double-blind, placebo-controlled evaluation of the use of diazepam after recent bereavement. Participants were randomised to either $2 \mathrm{mg}$ diazepam or identically packaged placebo up to three times daily. The primary outcome measure was the Bereavement Phenomenology Questionnaire.
\end{abstract}

\section{Results Thirty subjects were randomised. No evidence was found of an effect of benzodiazepines on the course of the first 6 months of bereavement (estimated mean difference of combined follow-up assessments $=0.3$ in favour of placebo; $95 \% \mathrm{Cl}-6.2$ to +6.7$)$.}

Conclusion We found no evidence of a positive or negative effect of benzodiazepines on the course of bereavement.

\section{Declaration of interest This study} was funded by the Sir Jules Thorn CharitableTrust.
The British National Formulary (British Medical Association, 2000) and the Committee on Safety of Medicines (1988) advise against using benzodiazepines after bereavement and suggest that these compounds may inhibit the grieving process. This advice appears to be based on anecdote; we are unaware of any controlled trials assessing the use of benzodiazepines in the management of bereavement. The current reluctance of clinicians and patients to use benzodiazepines is based on previous experience of misuse of these drugs. More rational prescribing of lower doses, for short periods, may be helpful in treating anxiety while avoiding risks of addiction. Future decisions about prescribing benzodiazepines should be driven by evidence of safety and efficacy based on this rational use. We aimed to clarify the role of benzodiazepines in the management of bereavement - specifically, whether receiving benzodiazepines at this time affected bereavement phenomenology - by testing the null hypothesis that there is no difference in treatment effect between diazepam and placebo on bereavement phenomenology.

\section{METHOD}

We undertook a randomised, double-blind, placebo-controlled, parallel-group trial of low-dose diazepam, prescribed within 2 weeks of bereavement of a spouse or partner.

\section{Ethics}

We were aware of the sensitive nature of this study, and ensured that only individuals who gave explicit informed consent were recruited. The local research ethics committee approved the study.

\section{Participants}

Recruitment took place over an 18-month period. We approached individuals whose partner had died at the Royal Free Hospital or the North London Hospice and who lived within a defined catchment area. Names and contact information of potential participants were provided by the bereavement officers within a couple of days of the death. All potential participants were sent a letter inviting them to participate in the study. Participants were then visited at home a few days later by a researcher (C.M. or J.W.).

Exclusion criteria were: possible alcohol dependence, indicated by a score of two or more on the CAGE questionnaire (Mayfield et al, 1974); a history of benzodiazepine dependence (defined by reported difficulty in stopping a previous course of benzodiazepines or related compounds); a current prescription of psychotropic medication; pregnancy or breast-feeding; severe respiratory, hepatic or renal disease; extensive cognitive impairment as reported by carers; difficulty in understanding English; and individuals over 60 with a history of falls.

\section{Intervention}

Participants were given a bottle of 20 tablets, which would be available to them for the following 6 weeks. Tablets were either $2 \mathrm{mg}$ diazepam or lactose placebo packaged identically. Participants were instructed that they could take up to 3 a day as required but that no further tablets would be available. Any remaining tablets were collected after 6 weeks. Bottle contents were randomised by the hospital pharmacy using a computerised schedule. Bottles were given out in numerical order. Success of the concealment of treatment allocation was assessed post-treatment by asking both participant and researcher to indicate their beliefs about the bottle contents on a $100 \mathrm{~mm}$ visual analogue scale with poles, 'definitely medication' and 'definitely not medication'. Individuals who were willing to participate in research but were unwilling to take medication were invited to complete questionnaires at all three time-points.

\section{Outcome measurement}

The primary outcome measure was the Bereavement Phenomenology Questionnaire (BPQ; Byrne \& Raphael, 1994). This is a 22item self-completed questionnaire rated on a four-point Likert scale. It seeks information about how often the respondent has been troubled by common and distressing aspects of bereavement in the previous 14 days. The maximum score is 66 . The higher the score, the greater the amount of bereavement phenomenology. The BPQ is reported to have good concurrent validity and internal consistency (Cronbach's $\alpha=0.83$ ) (Byrne $\&$ 
Raphael, 1994). The BPQ does not include any items about sleep disturbance, so four questions were extracted from an established sleep-quality questionnaire (Buysse et al, 1989) which covered the different types of disturbance that have been observed to occur during bereavement (Glick et al, 1974). These two questionnaires were completed at the point of recruitment, and then 8 weeks and 6 months after the bereavement. We chose not to use questionnaires specifically on symptoms of anxiety and depression because this was not germane to our primary research question. Furthermore, items about these phenomena are covered in the BPQ and we wished to keep our intrusion into the grief of the participants to a minimum.

Age, gender and occupation of the main wage earner in the household (pre-retirement occupation if applicable), the composition of the bereaved person's household and number of units of alcohol drunk in a typical week were recorded. At the end of the treatment period ( 6 weeks), the usefulness of the medication in reducing distress and helping the respondent to cope were recorded on visual analogue scales (using the poles 'not at all helpful' and 'extremely helpful') and a count was made of the number of tablets taken. At both follow-up meetings respondents were asked if they had received any help from other sources such as their general practitioner (GP), self-help groups such as Cruse, or from a bereavement counsellor.

\section{Statistics}

The intended sample size was 100 individuals in each treatment arm. This was calculated to be sufficient to allow detection of a standardised difference of 0.4 in the primary outcome and the post-treatment assessment with a significance level of $5 \%$ (two-sided) and power of $80 \%$. This difference was equivalent to a five-point change on the BPQ divided by the anticipated standard deviation of 11.9 (Byrne \& Raphael, 1994). This calculation was expected to overestimate the size of the required sample because we were unable to take account of the use of baseline measures in the analysis withoutanestimate ofthecorrelation between baseline and post-treatment assessments.

For the BPQ, the strength of evidence for any difference between the two treatment groups was assessed using an analysis of covariance with pre-treatment score as covariate and post-treatment and follow-up scores as repeated measures (Altman, 1991). This analysis gives two F-tests, one looking at the overall post-treatment difference (that is, combined results for the two follow-up points) between the two treatment groups, and the other looking at any evidence of a change in the relative effects of the two treatments between the two follow-up points. Data were analysed on observed cases (namely, participants who completed all three assessments) and on an intention-totreat basis. There were no differences in the conclusions drawn from the results of these two approaches to the analysis. Data on observed cases are presented in this paper.

\section{Non-randomised subjects}

Early in the trial it was observed that a large number of individuals who refused to be randomised nevertheless appeared eager to help with research in this area. We decided to invite these individuals to complete the same questionnaire battery at the same timepoints. We also introduced an additional questionnaire for completion by all participants at the final assessment. This was designed to elicit views pertinent to medical intervention during early bereavement. Summary statistics of the BPQ are presented for those non-randomised subjects completing all three assessment points. The results of the additional questionnaire are presented for those randomised in the main study and for the non-randomised group. Evidence for differences was evaluated using the MannWhitney $U$-test.

\section{RESULTS}

No adverse events were reported during the study and no participants requested further supplies of benzodiazepines.

\section{Recruitment}

Details of the number of exclusions and participants are given in Figure 1. The data resulting from the group of 40 nonrandomised subjects who completed all three assessments are presented alongside those from the two randomised groups.

A number of those people agreeing to be randomised were lost to follow-up either during the treatment period $(n=2)$ or during the follow-up period $(n=3)$, leaving 30 people who provided information at all three time points.

Baseline characteristics of the sample are shown in Table 1. A relatively large proportion of subjects was from social

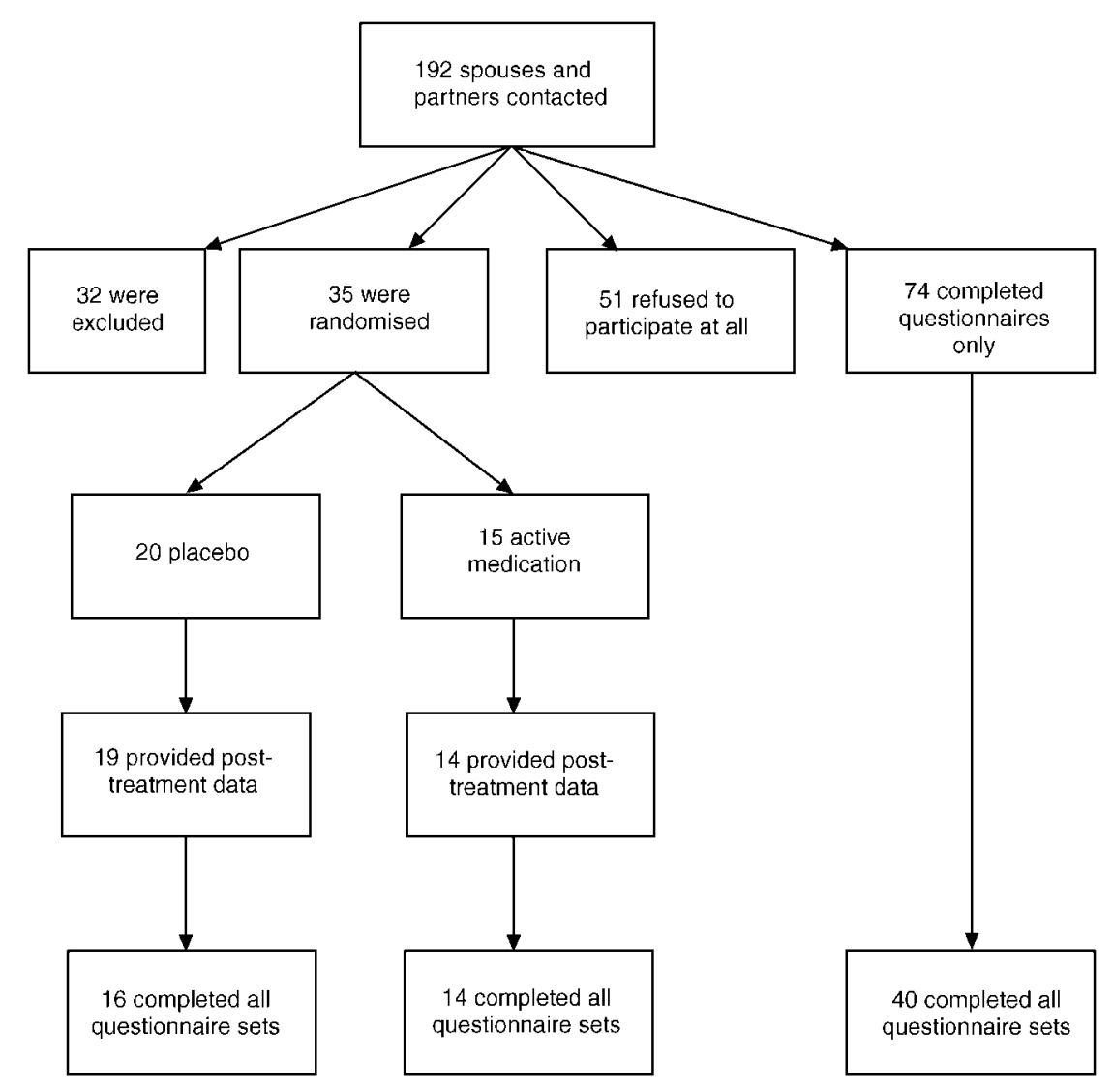

Fig. I Flow chart of recruitment to the study. 
classes I and II. There were no significant differences in baseline characteristics between the three groups.

The numbers of participants in the two randomised groups and the non-randomised group who had contact either with their GP or with a professional dedicated to bereavement work are shown in Table 2. Participants who declined to enter the medication arm of the study were less likely to consult their GP or a bereavement counsellor during the follow-up period (relative risk (RR) 0.42 , $95 \%$ CI $0.20-0.90)$. Those subjects who were randomised had comparable numbers of contacts with GPs. There was no significant difference between the two groups in the number of tablets taken by individuals. The median number of tablets taken by the whole sample was 6.5 tablets $(13 \mathrm{mg}$ diazepam) over the 6-week period (interquartile range 0.75-12.25).

\section{Bereavement phenomenology}

The mean overall BPQ score at first followup was 33.4 (s.d.=12.0). This is identical to the 6-week score reported by Byrne \& Raphael (1994). Table 3 gives the mean scores and standard deviations on the BPQ for the three groups at each of the three assessment points. In all three groups there is a small increase in the mean reported bereavement phenomenology immediately following the treatment period, with a decrease in the randomised groups at the 6-month follow-up. Comparing the two randomised groups, there is no evidence of the relative effects of diazepam and placebo changing over the post-treatment period $\left(F_{1,28}=0.39, P=0.54\right)$. Combining the data from the two post-treatment assessments, the estimated mean difference between the two groups was 0.3 in favour of placebo with a $95 \% \mathrm{CI}$ of -6.2 to +6.7 .

\section{Visual analogue scales}

There was no evidence to suggest that those people receiving active medication found it to be any more or less effective than placebo in reducing distress or in improving their coping. In each group an equal number of people fell above and below the overall medians for these two measures.

\section{Assessment of blinding}

Blinding was assessed in those participants who took at least 1 tablet. Nine of the 14 participants on placebo fell on the 'definitely not medication' side of the overall
Table I Baseline characteristics of sample

\begin{tabular}{|c|c|c|c|}
\hline & \multicolumn{3}{|c|}{ Group } \\
\hline & Active & Placebo & Non-randomised \\
\hline \multicolumn{4}{|l|}{ Variable } \\
\hline \multicolumn{4}{|l|}{ Gender } \\
\hline Male & 7 & 7 & 16 \\
\hline Female & 7 & 9 & 24 \\
\hline \multicolumn{4}{|l|}{ Social class } \\
\hline I & 5 & 7 & 7 \\
\hline II & 4 & 4 & 15 \\
\hline III & 3 & 3 & 14 \\
\hline IV & 1 & 2 & 1 \\
\hline V & 0 & 0 & 2 \\
\hline \multicolumn{4}{|l|}{ Living situation } \\
\hline Alone & II & II & 32 \\
\hline With family & 3 & 5 & 8 \\
\hline \multicolumn{4}{|c|}{ Typical weekly alcohol consumption' } \\
\hline Low & 10 & 14 & 39 \\
\hline Medium & 4 & I & I \\
\hline High & 0 & I & 0 \\
\hline Age, mean (s.d.) & $72(12)$ & $67(13)$ & $68(14)$ \\
\hline \multirow{2}{*}{\multicolumn{4}{|c|}{$\begin{array}{l}\text { Overall quality of sleep in previous week } \\
\text { at baseline }\end{array}$}} \\
\hline & & & \\
\hline Very good & 3 & 0 & 9 \\
\hline Good & 6 & 8 & 19 \\
\hline Poor & 5 & 7 & 7 \\
\hline Very poor & 0 & I & 3 \\
\hline
\end{tabular}

I. For males: $L o w=0-20$, Medium $=21-39$, High $=40+$ units of alcohol. For females: $L o w=0-13$, Medium $=14-39$, $\mathrm{High}=40+$ units of alcohol. median, and $6 / 8$ on active medication (one missing value) fell on the 'definitely medication' side of the overall median. Subjects were not aware of their treatment allocation (Fisher's exact test $P=0.18$ ). There was no evidence that the interviewers were aware of the treatment allocation.

\section{Sleep problems}

Table 4 gives changes observed in the responses given on the sleep questionnaire from pre- to post-treatment and from posttreatment to the end of follow-up. Overall there is little evidence of a treatment effect
Table 2 Contact with professionals during the treatment period and the follow-up period

\begin{tabular}{lllr}
\hline Variable & \multicolumn{3}{c}{ Group } \\
\cline { 2 - 4 } & Active & Placebo & Non-randomised \\
\hline Contacts during treatment period & 9 & 10 & 32 \\
None & 3 & 5 & 4 \\
General practitioner & 2 & 1 & 4 \\
Bereavement professional & & & 26 \\
Contacts during follow-up period & 7 & 8 & 3 \\
None & 4 & 5 & 4 \\
General practitioner & 3 & 3 & \\
Bereavement professional & & & \\
\hline
\end{tabular}


Table 3 Mean scores (standard deviations) for the Bereavement Phenomenology Questionnaire

\begin{tabular}{lccc}
\hline Time point & \multicolumn{3}{l}{ Group } \\
\cline { 2 - 4 } & $\begin{array}{c}\text { Active } \\
(n=14)\end{array}$ & $\begin{array}{c}\text { Placebo } \\
(n=16)\end{array}$ & $\begin{array}{c}\text { Non-randomised } \\
(n=40)\end{array}$ \\
\hline 2 weeks & $30.43(13.39)$ & $33.69(9.41)$ & $34.35(13.96)$ \\
8 weeks & $31.71(13.89)$ & $34.31(10.12)$ & $32.85(13.41)$ \\
7 months & $28.00(9.15)$ & $28.75(12.75)$ & $32.98(15.36)$ \\
\hline
\end{tabular}

in reported sleep quality. There is some indication that those individuals receiving placebo showed greater improvement at the end of the treatment period in being able to get to sleep quickly $(P=0.06)$ and in having fewer bad dreams $(P=0.04)$.

Results of the questionnaire regarding beliefs about treatment after bereavement are shown in Table 5. A number of significant differences emerged, indicating that concerns about the efficacy of medication

Table 4 Change in the frequency of sleep problems were associated with the decision not to participate in the study.

In the years after their development, benzodiazepines were frequently prescribed to give symptomatic relief for distress after bereavement; surveys conducted in the 1960 s showed that $25-50 \%$ of people recently

\section{DISCUSSION}

\begin{tabular}{|c|c|c|c|}
\hline & \multicolumn{2}{|c|}{ Group } & \multirow[t]{2}{*}{$P$} \\
\hline & Active & Placebo & \\
\hline \multicolumn{4}{|l|}{ From week 2 to week 8} \\
\hline Could not get to sleep within 30 minutes of going to bed & & & 0.06 \\
\hline Got worse/stayed bad & 9 & 4 & \\
\hline Got better/stayed good & 5 & 12 & \\
\hline Woke early, could not get back to sleep & & & 0.73 \\
\hline Got worse/stayed bad & 8 & 8 & \\
\hline Got better/stayed good & 6 & 8 & \\
\hline Bad dreams & & & 0.04 \\
\hline Got worse/stayed bad & 4 & 0 & \\
\hline Got better/stayed good & 10 & 16 & \\
\hline Rating of overall sleep quality & & & 0.44 \\
\hline Got worse/stayed bad & 6 & 4 & \\
\hline Got better/stayed good & 8 & 12 & \\
\hline \multicolumn{4}{|l|}{ From week 2 to month 7} \\
\hline Cannot get to sleep within 30 minutes of going to bed & & & 0.42 \\
\hline Got worse/stayed bad & 5 & 3 & \\
\hline Got better/stayed good & 9 & 13 & \\
\hline Woke early, could not get back to sleep & & & 0.26 \\
\hline Got worse/stayed bad & 3 & 7 & \\
\hline Got better/stayed good & II & 9 & \\
\hline Bad dreams & & & 1.00 \\
\hline Got worse/stayed bad & $\mathrm{I}$ & 2 & \\
\hline Got better/stayed good & 14 & 13 & \\
\hline Rating of overall sleep quality & & & 1.00 \\
\hline Got worse/stayed bad & I & I & \\
\hline Got better/stayed good & 13 & 15 & \\
\hline
\end{tabular}

bereaved of a spouse were prescribed tranquillisers (Parkes, 1964; Parkes \& Weiss, 1983). A more recent survey showed much less use of benzodiazepines, raising the possibility that bereaved individuals are denied help in coping with their grief (Warner \& King, 1997).

Two main issues arising from this study will be considered; first, the implications of the results, and second, exploration of the difficulty we experienced with recruitment and the impact that this may have on future research in this area.

\section{Randomised subjects}

We found no evidence that diazepam affected the course of bereavement, and respondents were neither more nor less likely to find diazepam helpful in reducing distress or increasing coping compared with placebo. While the intended sample size was not achieved, it is likely that this was overestimated. Although this is a relatively small study, the results are useful, as clinically significant effect sizes can still be detected with precision. We recruited sufficient numbers to detect a nine-point difference in the BPQ scores (0.75 s.d.), with $80 \%$ power, and the numbers of subjects recruited to this study do provide useful information on the effect of benzodiazepines after bereavement. Our results suggest that the estimated mean difference between the two groups on the BPQ was less than one point, and the $95 \%$ confidence interval indicates that a difference between the two groups of greater than seven points is unlikely. Changes of such small magnitude are unlikely to be clinically significant (a reduction of seven points being equivalent to a change from 'often experienced' to 'sometimes experienced' for seven of the 22 items). Thus benzodiazepines may have a neutral effect in the course of bereavement, and are unlikely to have a major impact on it in the doses used in this study. There was, however, some evidence that those subjects who received diazepam appeared less likely to have resolution of sleep problems in the weeks after bereavement.

The absence of a treatment effect may be related to the relatively low doses of diazepam taken in this study. However, we intended to prescribe benzodiazepines in a way that reflects recommended practice in primary care - namely, relatively low doses, used as required over a short period of time (Ashton, 1994). The absence of an effect may also be due, in part, to the small number of tablets 
Table 5 Views on treatment after bereavement

\begin{tabular}{|c|c|c|c|c|c|c|}
\hline \multirow[t]{2}{*}{ Statement } & \multicolumn{6}{|c|}{ Response } \\
\hline & Strongly agree & Agree & Undecided & Disagree & Strongly disagree & $P$ \\
\hline If a person is not sleeping well after a bereavement, tablets could be helpful & & & & & & 0.0004 \\
\hline Randomised & 6 & 16 & 6 & I & I & \\
\hline Non-randomised & I & 13 & 12 & 7 & 6 & \\
\hline My friends and family have not been able to provide the support I needed & & & & & & 0.29 \\
\hline \multicolumn{7}{|l|}{ during my bereavement } \\
\hline Randomised & 0 & I & 2 & 8 & 19 & \\
\hline Non-randomised & 1 & 5 & 2 & 10 & 21 & \\
\hline Generally, I avoid taking tablets when possible & & & & & & 0.19 \\
\hline Randomised & 17 & 12 & I & 0 & 0 & \\
\hline Non-randomised & 16 & 17 & 0 & I & 3 & \\
\hline Tablets may be helpful for some people during bereavement but I & & & & & & 0.61 \\
\hline \multicolumn{7}{|l|}{ was able to manage without } \\
\hline Randomised & 10 & II & 5 & 4 & 0 & \\
\hline Non-randomised & 12 & 20 & 5 & I & I & \\
\hline Tablets may help a person cope after a bereavement but they & & & & & & 0.11 \\
\hline \multicolumn{7}{|l|}{ delay coming to terms with the loss } \\
\hline Randomised & 6 & II & 7 & 5 & I & \\
\hline Non-randomised & 8 & 21 & 9 & 0 & 0 & \\
\hline An emotionally strong person may still find that tablets help & & & & & & 0.004 \\
\hline \multicolumn{7}{|l|}{ coping with grief } \\
\hline Randomised & 4 & 19 & 4 & 1 & 2 & \\
\hline Non-randomised & I & 15 & 12 & 10 & I & \\
\hline It is better to use willpower to cope with grief than resort to & & & & & & 0.02 \\
\hline \multicolumn{7}{|l|}{ medication } \\
\hline Randomised & 5 & 12 & 3 & 9 & I & \\
\hline Non-randomised & 13 & 17 & 7 & 1 & I & \\
\hline Tablets taken to help with sleep are addictive & & & & & & 0.14 \\
\hline Randomised & 5 & 10 & 10 & 3 & 2 & \\
\hline Non-randomised & 5 & 22 & 10 & I & 0 & \\
\hline Tablets would be of no help to a person who found themselves to & & & & & & 0.004 \\
\hline \multicolumn{7}{|l|}{ be very tense and anxious after a bereavement } \\
\hline Randomised & 2 & 4 & 5 & 14 & 5 & \\
\hline Non-randomised & I & 12 & 18 & 7 & I & \\
\hline Tablets should be given to a bereaved person only after & & & & & & 0.63 \\
\hline \multicolumn{7}{|l|}{ they have seen a doctor } \\
\hline Randomised & II & 16 & 0 & 3 & 0 & \\
\hline Non-randomised & 16 & 20 & 3 & 0 & 0 & \\
\hline I would have liked to have been offered the tablets sooner after & & & & & & 0.06 \\
\hline \multicolumn{7}{|l|}{ the death } \\
\hline Randomised & I & 2 & 7 & 14 & 6 & \\
\hline Non-randomised & 0 & I & 5 & 18 & 13 & \\
\hline
\end{tabular}

taken. The reason for this may have been either a perceived lack of need or reluctance, even among this group, to use medication.

No subjects who took benzodiazepines in this study developed dependence. Although tolerance and dependence are potential problems when prescribing benzodiazepines, rational prescribing of long-acting preparations such as diazepam, in small doses for limited periods, is gaining acceptance (Woods \& Winger, 1995).

\section{Non-randomised subjects}

Many individuals were willing to complete the questionnaires, but unwilling to be randomised. There were significant differences between these two groups. Subjects willing to be randomised had more conviction about the utility of tranquillisers. This does not hamper the validity of the study, in that the study sample reflects those individuals more likely to request and accept medication after 
bereavement. Some individuals may have been reluctant to accept medical intervention for their bereavement, suggested by the trend for non-randomised subjects to visit their GP less often. Others declined to participate in this study because they were antipathetic to the concept of taking medication, especially tranquillisers, after bereavement. Therefore, a broader evaluation of this question may not be possible.

There are several explanations for the low recruitment to the randomised arms of this study other than cynicism about the efficacy of the medication. Many individuals who were approached did not report any feelings of grief (18 of the 70 subjects scored 22 or less on the BPQ) and did not perceive the need for medication. There were also some logistical problems in recruitment. Partners in one local multipartner practice refused to allow their patients to participate because of the perceived harm benzodiazepines would do to them. Some potential subjects moved to relatives after the bereavement and could not be recruited. Family members sometimes opposed participation in the trial, despite the individual's willingness.

A relatively high proportion of the randomised sample were drawn from social classes I and II, although this was not the case in the non-randomised group. This may suggest that individuals from higher social classes are more willing to participate in research of this nature. We recruited subjects whose partner had died in a hospital or hospice. Individuals who experience death of a partner at home may have a different bereavement process, with less anticipatory bereavement and professional support. We did consider also recruiting from general practices, but decided to restrict the trial to deaths in hospital because of the logistical difficulties in identifying subjects quickly enough.

Research on individuals who have recently been bereaved may be perceived as insensitive or intrusive. In common with our previous experience, it appears that bereaved individuals do not share this view (Warner \& King, 1997). Very few of the bereaved individuals we contacted were hostile to the approach and most were willing to participate in research, at least in filling in the questionnaire. Furthermore, follow-up of those randomised was very high; only one subject withdrew consent after randomisation.

Many of the problems we encountered with this study were based on an

\section{CLINICAL IMPLICATIONS}

- The assumption that benzodiazepines inhibit the grieving process should be reconsidered.

- Diazepam may have a neutral effect on the course of bereavement.

- Most bereaved subjects welcome research in this area.

\section{LIMITATIONS}

Because of the small numbers in this study, only relatively large treatment effects can be detected with precision.

- The sample was selective, although it probably represents those individuals willing to take tranquillisers after bereavement.

Relatively low doses of diazepam may have attenuated the treatment effect.

JAMES WARNER, MD, Imperial College School of Medicine, St Charles Hospital, London; CHRIS METCALFE, MSc, Department of Medical Statistics and Evaluation, Imperial College School of Medicine, Hammersmith Hospital, London; MICHAEL KING, PhD, Department of Psychiatry and Behavioural Sciences, The Royal Free and University College Medical School, Royal Free Campus, London

Correspondence: Dr James Warner, Senior Lecturer in Old Age Psychiatry, Imperial College School of Medicine, Exmoor Street, London WI0 6DZ,UK. Tel: 0208962 4I05; fax: 0208960 8II2; e-mail: j.warner@ic.ac.uk

(First received 17 January 2000, final revision 30 May 2000, accepted 19 June 2000)

uninformed opinion of potential subjects and some doctors, resulting in reluctance to participate because of pre-existing concerns about benzodiazepines. While the antipathy to this class of drugs persists, it may never be possible to prove beyond doubt whether or not they are harmful or helpful.

\section{ACKNOWLEDGEMENTS}

We thank Angela Thomas for help with data collection and the Pharmacy Department at the Royal Free Hospital for assistance with the randomisation and blinding. We also thank the staff of the Bereavement Office at the Royal Free Hospital and the staff of the North London Hospice for their help in contacting potential participants.

\section{REFERENCES}

Altman, D. G. (1991) Practical Statistics for Medical Research. London: Chapman \& Hall.

Ashton, H. (1994) Guidelines for the rational use of benzodiazepines - when and what to use. Drugs, $\mathbf{4 8}$ $25-40$.

British Medical Association \& Royal Pharmaceutical Society of Great Britain (2000)
British National Formulary (September). London \& Wallingford: BMJ Books \& Pharmaceutical Press.

Buysse, D. J., Reynolds, C. F., Monk, T. H., et al (1989) The Pittsburgh sleep quality index. A new instrument for psychiatric practice and research. Psychiatric Research, 28, 193-213.

Byrne, G. J. A. \& Raphael, B. (1994) A longitudinal study of bereavement phenomena in recently widowed elderly men. Psychological Medicine, 24, 4II-42I.

Committee on Safety of Medicines (1988) Current Problems, No. 21.

Glick, I. O., Weiss, R. S. \& Parkes, C. M. (1974) The First Year of Bereavement. New York: Wiley.

Mayfield, D., McLeod, G. \& Hall, P. (1974) The CAGE questionnaire: validation of a new alcoholism instrument. American Journal of Psychiatry, |3 |, ||2|-||23.

Parkes, C. M. (1964) Effects of bereavement on physical and mental health: a study of the medical records of widows. British Medical Journal, 2, 274-279.

—\& Weiss, R. S. (1983) Recovery from Bereavement. New York: Basic Books.

Warner, J. P. \& King, M. B. (1997) The use of benzodiazepines after bereavement. Bereavement Care, 16, $14-15$.

Woods, J. H. \& Winger, G. (1995) Current benzodiazepine issues. Psychopharmacology, II8, 107-1I5. 